\title{
Brain Activation During Autobiographical Memory Retrieval with Special Reference to Default Mode Network
}

\author{
Tadashi Ino, ${ }^{\mathrm{a}, *}$, Ryusuke Nakai ${ }^{\mathrm{b}}$, Takashi Azuma ${ }^{\mathrm{b}}$, Toru Kimura a and Hidenao Fukuyama ${ }^{\mathrm{c}}$
}

\author{
${ }^{a}$ Department of Neurology, Rakuwakai-Otowa Hospital, Otowachinjicho 2, Yamashina-ku, Kyoto 607-8062, Japan; \\ ${ }^{b}$ Research Center for Nano Medical Engineering, Institute for Frontier Medical Sciences, Kyoto University. Shogoin, \\ Sakyo-ku, Kyoto 606-8507, Japan; ${ }^{c}$ Department of Brain Pathophysiology, Human Brain Research Center, Graduate \\ School of Medicine, Kyoto University, Shogoin, Sakyo, Kyoto, 606-8507, Japan
}

\begin{abstract}
Recent neuroimaging studies have suggested that brain regions activated during retrieval of autobiographical memory $(\mathrm{ABM})$ overlap with the default mode network (DMN), which shows greater activation during rest than cognitively demanding tasks and is considered to be involved in self-referential processing. However, detailed overlap and segregation between $\mathrm{ABM}$ and DMN remain unclear. This fMRI study focuses first on revealing components of the DMN which are related to $\mathrm{ABM}$ and those which are unrelated to $\mathrm{ABM}$, and second on extracting the neural bases which are specifically devoted to ABM. Brain activities relative to rest during three tasks matched in task difficulty assessed by reaction time were investigated by fMRI; category cued recall from ABM, category cued recall from semantic memory, and number counting task. We delineated the overlap between the regions that showed less activation during semantic memory and number counting relative to rest, which correspond to the $\mathrm{DMN}$, and the areas that showed greater or less activation during $\mathrm{ABM}$ relative to rest. $\mathrm{ABM}$-specific activation was defined as the overlap between the contrast of $\mathrm{ABM}$ versus rest and the contrast of ABM versus semantic memory. The fMRI results showed that greater activation as well as less activation during $\mathrm{ABM}$ relative to rest overlapped considerably with the DMN, indicating that the DMN is segregated to the regions which are functionally related to $\mathrm{ABM}$ and the regions which are unrelated to ABM. ABM-specific activation was observed in the left-lateralized brain regions and most of them fell within the DMN.
\end{abstract}

Keywords: Autobiographical memory, semantic memory, default mode network, functional MRI.

\section{INTRODUCTION}

Autobiographical memory retrieval (ABM) is the conscious reconstruction and recollection of a personally relevant event [1]. The neuroanatomical basis of the ABM has been consistently reported to be located in the retrosplenial/posterior cingulate cortex, medial temporal lobe including hippocampus, medial prefrontal cortex (PFC), and the inferior parietal lobule $[2,3]$. These regions have also been reported to be the components of the default mode network (DMN) where increased activity during rest or lowdemand task compared with high-demand tasks is observed $[4,5,6]$. Although the function of the DMN is unclear, it is postulated that the DMN is involved in various cognitive processes such as stimulus independent thoughts (i.e. cognitive processes that are not related to any information in the immediate external environment) or mind-wandering [7], self-referential sensory processing of interoceptive sensory information and scanning the external environment in the absence of attention-demanding stimuli [8-10], and suppression of the endogenous activity in limbic and paralimbic systems [11].

\footnotetext{
*Address correspondence to this author at the Department of Neurology, Rakuwakai-Otowa Hospital, Otowachinjicho 2, Yamashina-ku, Kyoto 6078062, Japan; Tel: +81-75-593-4111; Fax: +81-75-581-6935

E-mail: rakuwadr042@rakuwadr.com
}

According to Andreasen et al. [12], typical mental activity during rest consists of a mixture of freely wandering past recollections, future plans, and other personal thoughts. It has been proposed that prominent part of the processes mediated by the DMN is episodic retrieval [8, 13], and recent studies showed that common regions are involved in the ABM and DMN $[14,15]$. However, the DMN has been suggested to be involved in functions other than ABM such as self-referential sensory processing of interoceptive sensory information $[8,9]$ and social cognition $[14,15,16]$. A recent meta-analysis using a combination of activation likelihood estimation suggested that the DMN can be dissociable to different subnetworks, each with distinct function [17]. An fMRI study using Granger causality analysis also suggested heterogeneity within the DMN [18].

The present study aimed to reveal components of the DMN which are related to $\mathrm{ABM}$ and those which are unrelated to ABM. The former will be shown as the overlap between greater activation during ABM and the DMN. Since $\mathrm{ABM}$ is also attention-demanding and goal-directed task, parts of the regions which showed less activation during ABM relative to rest will be involved in the DMN. Therefore, DMN which are unrelated to ABM will be shown as the overlap between less activation during ABM relative to rest and the DMN. No previous study has addressed the anatomical overlap between the DMN and less activation for ABM. In the present study, we investigated the extent to which less activation as well as greater activation during ABM relative 
to rest overlaps with the DMN. In order to identify the DMN, we used number counting task and semantic memory retrieval task (see methods section). Number counting task requires a type of stimulus-dependent thought and therefore less activation during this task relative to rest will delineate a typical DMN. Although semantic memory retrieval task is defined as the conscious recollection of information about the world [19], it requires a search for and selection of appropriate items which may have a certain level of personal relevance [20]. Therefore, less activation during semantic memory retrieval will involve relatively limited parts of the DMN. However, we selected this task since we are also interested in isolating the brain areas which uniquely contribute to ABM and assumed that these areas can be estimated by the combination of greater activation during $A B M$ relative to rest and greater activation during $A B M$ relative to semantic memory due to the following consideration. Previous studies regarding the neural substrates of ABM have measured brain activities during $\mathrm{ABM}$ relative to reference task such as rest or retrieval of semantic memory [3]. However, the regions which showed greater activation during $A B M$ relative to rest will include the generative cognitive processes which are not specifically related to ABM, such as attention and response execution, which will be removed by the contrast of ABM vs. semantic memory. On the other hand, the areas which showed greater activation during $\mathrm{ABM}$ relative to semantic memory will be contaminated by the region which showed reduced activation during semantic memory relative to rest but no activation during ABM relative to rest. This contamination will be removed by the contrast of $\mathrm{ABM}$ vs. rest. Therefore, the overlap between the contrast of $\mathrm{ABM}$ vs. rest and the contrast of $\mathrm{ABM}$ vs. semantic memory can be regarded as relatively conservative estimate of brain activation unique to ABM.

\section{METHODS}

\section{Subjects}

Twenty one volunteers (10 males and 11 females), aged 20-35 (mean \pm SD; $25.7 \pm 5.3$ ) participated in this study. All participants were right-handed and their scores for the Japanese version of the Edinburgh handedness inventory [21] were all above +80 . All participants were free of any psychiatric or neurological illnesses. The experimental procedure was approved by the Medical Ethics Committee of Otowa Hospital, and all participants gave their written informed consent.

\section{Tasks}

There were four conditions in the fMRI examination; ABM, semantic memory retrieval, number counting, and rest. During fMRI, 7 epochs of rest, 6 epochs of each task condition (ABM, semantic memory and number counting), each of which lasted $16 \mathrm{~s}$, were presented in the pseudorandom order with a constraint that both the first and the last conditions were rest and never the same condition in a row, and the order was counterbalanced across subjects. The tasks of ABM, semantic memory, and number counting were constructed on the basis of an independent behavioral study on reaction times (RTs) so that averaged RTs were similar between three tasks. Subjects pressed a button whenever they recalled one item which belongs to the assigned category in the cue sentence during $\mathrm{ABM}$ and semantic memory, and whenever they counted the even number during number counting. During ABM, the subjects were presented with the following six cue statements one by one for each epoch (1) the food which you have eaten, (2) the person to whom you have a conversation, (3) the television program which you have watched, (4) the place where you have eaten out, (5) the magazine or book you have read, and (6) the place where you did shopping. They were instructed to travel backward from the present moment in order to produce items which belongs to each cue category (e.g. food) as much as possible and press a button with their right index finger each time when one appropriate item came to mind. During semantic memory task the subjects were presented with the following six cue statements one by one for each epoch; (1) bird, for example, pigeon, (2) flower, for example, tulip, (3) carpenter tool, for example, hammer, (4) quadrupedal animal, for example, zebra, (5) tree, for example, pine, and (6) insect, for example, dragonfly. They were instructed to produce items which belong to each cue category (e.g. bird) as much as possible and required to press a button with their right index finger each time when one appropriate item came to mind. During number counting, subjects were presented visually with the statement "push button when you count even numbers, 1111, 1112" (different two successive numbers of four digits were given for each epoch). They were required to count mentally the following number sequentially $(1113,1114,1115,1116,---)$ and press a button with their right index finger each time when the subjects counted the even number $(1114,1116)$. During rest condition, subjects were given the statement "try to empty your mind as best as you can" and required to think as little as possible. These cue statements were projected onto a transparent screen, using a personal computer with custom written software and a projector, which the participants viewed through a mirror attached to a head coil. Each cue statement remained on the screen for $16 \mathrm{sec}$ until the next cue was presented. The visual images of the cue statements were balanced across conditions for numbers of characters included in each statement $(12.8 \pm 1.7$ for ABM, $12.8 \pm 2.3$ for semantic memory, 13 for number counting and rest). The participants were given a full explanation of the experimental details and performed a short version of the task with cue statements not used in the fMRI experiment (e.g. "the place where you had a trip" for ABM, and "fish, for example, tuna" for semantic memory task) in the scanner before real scanning.

\section{Image Acquisition and Analysis}

Gradient echo, echo-planar images $(\mathrm{TR}=2 \mathrm{~s}, \mathrm{TE}=50$ $\mathrm{ms}$, flip angle $=90^{\circ}, \mathrm{FOV}=200 \mathrm{~mm}$, matrix $=64 \times 64$, slice thickness $=4 \mathrm{~mm}$, gap $=1 \mathrm{~mm}, 25$ axial slices) and a three-dimensional $\mathrm{T} 1$ weighted image for anatomical normalization $\left(\mathrm{TR}=11.6 \mathrm{~ms}, \mathrm{TE}=4.9 \mathrm{~ms}\right.$, flip angle $=8^{\circ}$, $\mathrm{FOV}=220 \times 220 \mathrm{~mm}$, matrix $=256 \times 256$, slice thickness $=$ $1 \mathrm{~mm}$ ) were obtained using a $1.5-\mathrm{T}$ Siemens Sonata. The first 5 scans were excluded and the remaining fMRI data were analyzed using SPM5. After slice timing correction and realignment, all images were coregistered to the T1 weighted image and spatially normalized (voxel size $2 \times 2 \times 2 \mathrm{~mm}$ ) to the T1 template in the MNI space, and smoothed with a 7 mm FWHM Gaussian filter. High pass temporal filtering with $128 \mathrm{~s}$ was applied and serial autocorrelations were 
modeled with the AR (1) model in SPM5. Motion parameters defined by the realignment procedure were added to the model as six regressors of no interest. The data of each condition (ABM, semantic memory, number counting, and rest) were modeled by a boxcar convolved with a canonical hemodynamic response function (HRF). The numbers of responses during each task period ( $\mathrm{ABM}$, semantic memory, and number counting) were included as a linear parametric modulation in order to remove the effects related to the number of responses. The contrasts of each task condition compared with rest were computed. Parameter estimates of these contrasts for each subject were subjected to a flexible factorial ANOVA design in SPM5. The first interest of the present study was to examine which parts of activation and which parts of less activation during ABM were overlapped with the DMN. For this purpose, greater activation during $\mathrm{ABM}(\mathrm{ABM}>$ rest) and less activation during $\mathrm{ABM}$ (ABM $<$ rest) were overlaid on less activation during semantic memory (semantic memory < rest) and on less activation during number counting (number counting < rest). The second purpose was to extract the brain regions which are specifically related to ABM. For this purpose, we delineated the overlap between the contrast of $A B M$ vs. rest and the contrast of $\mathrm{ABM}$ vs. semantic memory due to a reason described in the introduction. The statistical threshold was set at $p<0.05$, corrected for multiple comparisons using false discovery rate, and a cluster extent threshold of 20 contiguous voxels.

\section{RESULTS}

\section{Behavioral Results}

The numbers of button presses (mean \pm SD) during one epoch (16 s) for each task condition was $6.8 \pm 1.4$ for ABM, $6.5 \pm 1.5$ for semantic memory, and $6.4 \pm 1.4$ for number counting. The RTs was $2.4 \pm 0.46 \mathrm{~s}$ for ABM, $2.6 \pm 1.1 \mathrm{~s}$ for semantic memory, and $2.7 \pm 0.72 \mathrm{~s}$ for number counting. Those data were subjected to a repeated measures ANOVA. There was no significant difference between the three conditions for numbers of button presses $(\mathrm{F}(2,19)=0.9, p=$ $0.40)$ and for RTs $(\mathrm{F}(2,19)=1.9, p=0.18)$.

\section{FMRI RESULTS}

The brain regions which showed greater activation during ABM compared with other condition, namely, rest, semantic memory, and number counting are shown in Fig. (1). The brain regions which showed less activation during ABM, semantic memory, and number counting, relative to rest, are shown in Fig. (2). In order to visualize which parts of greater activation and less activation during $\mathrm{ABM}$ relative to rest overlapped with the DMN, they were shown simultaneously with the regions which showed less activation during semantic memory and number counting relative to rest. These maps are shown in Fig. (3), where the overlap between greater activation during $\mathrm{ABM}(\mathrm{ABM}>$ rest) and less activation during semantic memory (semantic memory < rest) or number counting (number counting < rest) are shown in yellow color, and the overlap between less activation during $\mathrm{ABM}(\mathrm{ABM}<\mathrm{rest})$ and less activation during semantic memory or number counting are shown in light blue. There was a high level of overlap between greater activation during $\mathrm{ABM}$ and less activation during number counting; extensive areas including bilateral posterior cingulate gyrus, left dorsal premotor area, left ventromedial PFC, left hippocampal region, left inferior parietal lobule, left middle temporal gyrus, and left inferior frontal gyrus showed greater activation during $\mathrm{ABM}$ and less activation during number counting (Fig. 3, Table 1b). However, there was a relatively little overlap between greater activation during $\mathrm{ABM}$ and less activation during semantic memory; bilateral posterior cingulate gyri, left precuneus, left ventrolateral PFC, and left inferior parietal lobule showed greater activation during $\mathrm{ABM}$ and less activation during semantic memory (Fig. 3, Table 1a). This is consistent with our prediction that less activation during semantic memory will reveal a limited part of the $\mathrm{DMN}$, since semantic memory is related to a certain level of personal relevance. A sizable portion of the less activation during ABM overlapped with less activation during both semantic memory and number counting; extensive brain regions including bilateral paracentral lobules extending to the anterior part of the precuneus, bilateral inferior parietal lobules, bilateral posterior insula extending to the superior temporal gyri, right
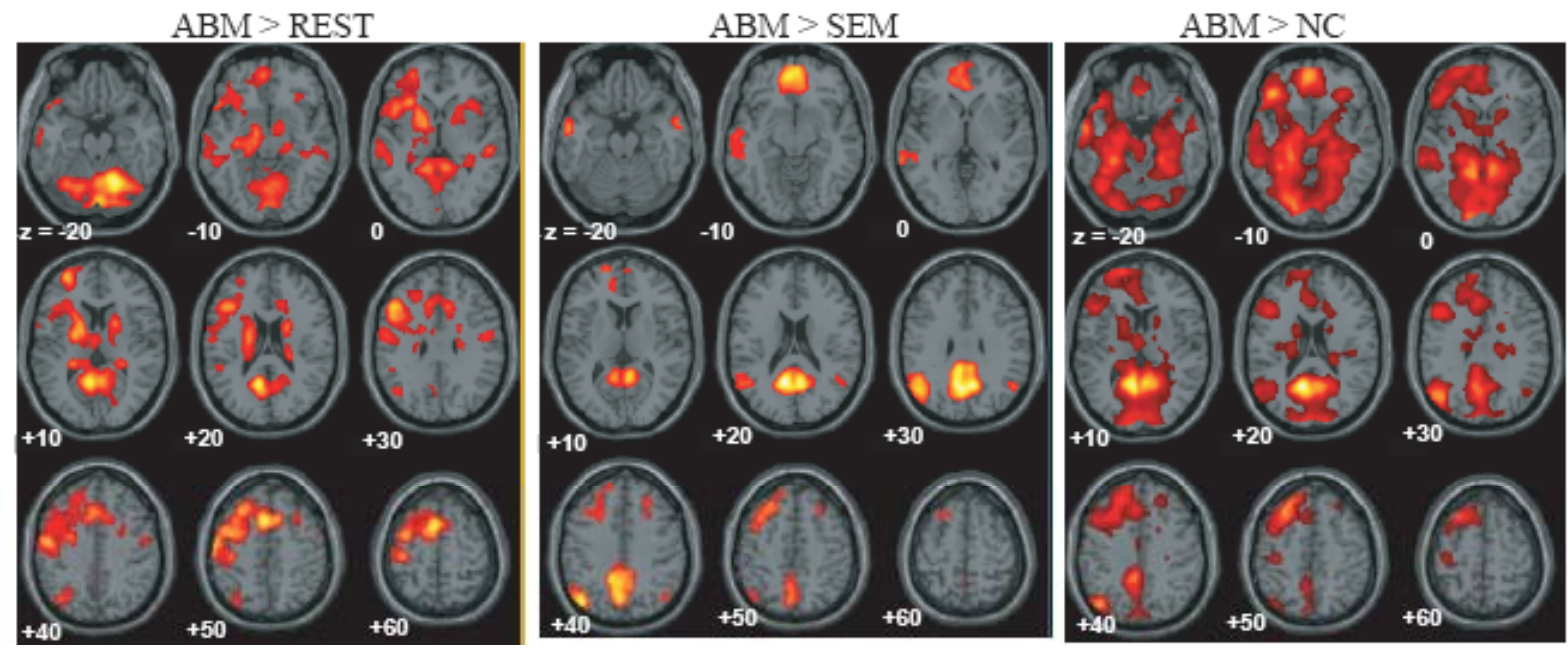

Fig. (1). Regions which showed greater activation during ABM relative to rest, semantic memory and number counting, superimposed on the axial images of the MNI T1 template. SEM = semantic memory; REST $=$ rest. $\mathrm{NC}=$ number counting. 

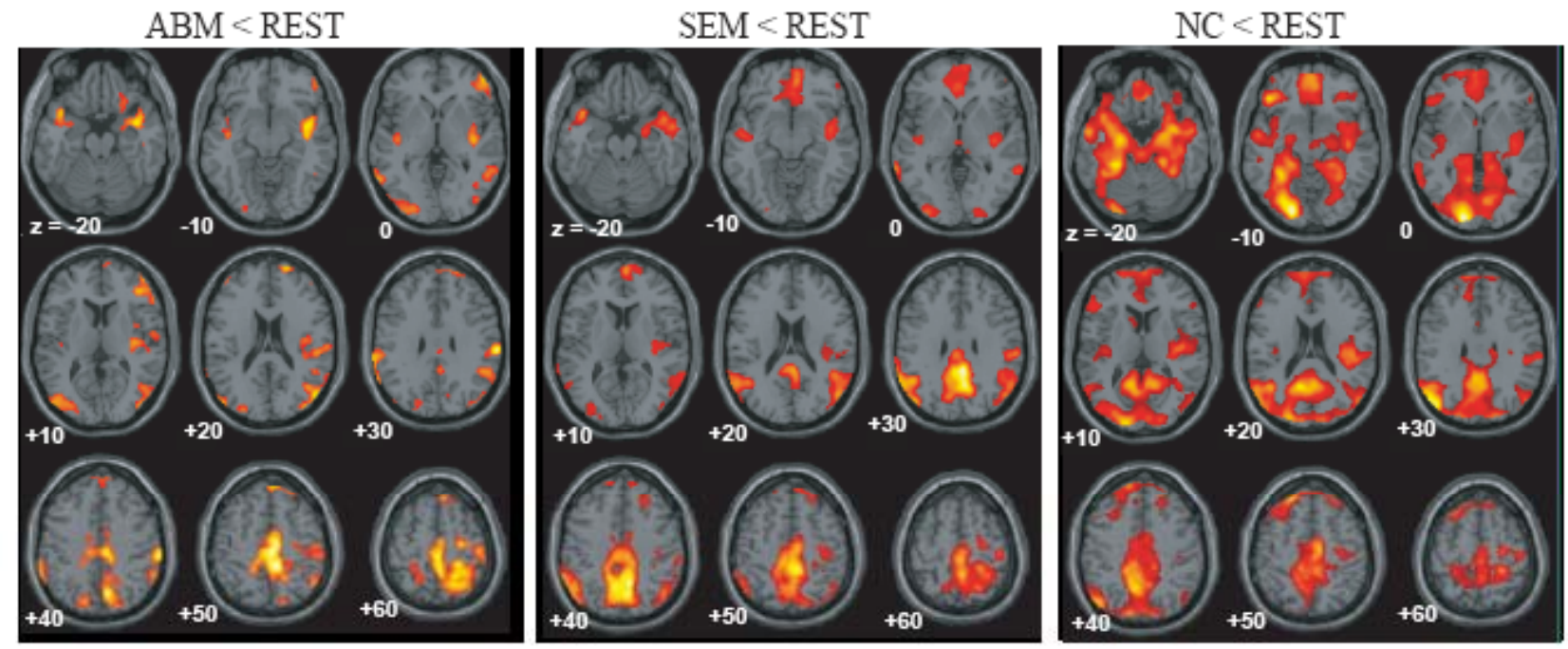

Fig. (2). Regions which showed less activation during ABM, semantic memory and number counting, superimposed on the axial images of the MNI T1 template.
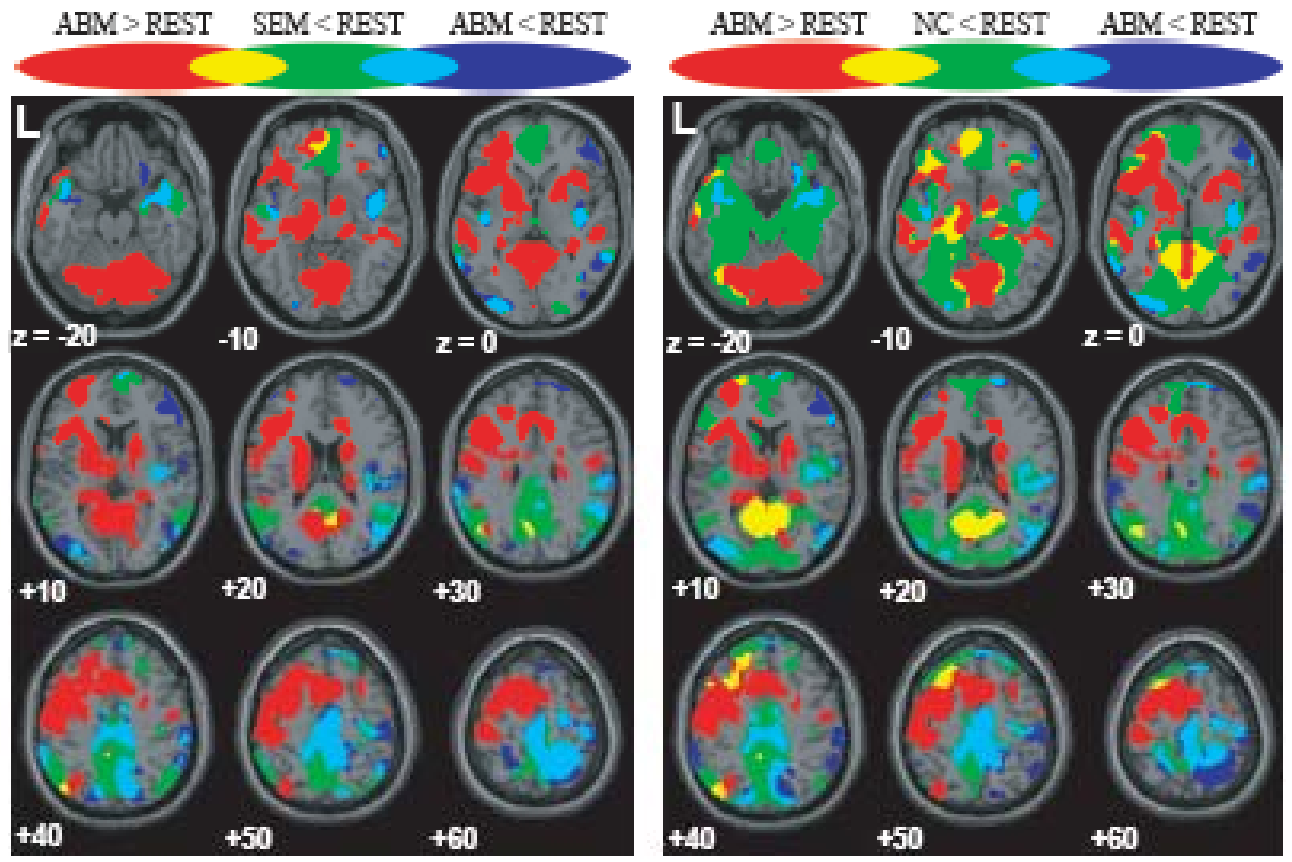

Fig. (3). Regions which showed greater/less activation during ABM in relation to the DMN, superimposed on the axial images of the MNI T1 template. red $=\mathrm{ABM}>\mathrm{REST}$; blue $=\mathrm{ABM}<\mathrm{REST}$; green $=\mathrm{SEM}<\mathrm{REST}$ or NC $<$ REST; yellow = overlap between ABM $>$ REST and SEM $<$ REST or that between ABM $>$ REST and NC $<$ REST; light blue = overlap between ABM $<$ REST and SEM $<$ REST or that between $\mathrm{ABM}<$ REST and NC $<$ REST.

premotor area, right ventrolateral PFC, and right frontopolar cortex showed less activation during ABM, semantic memory, and number counting (Fig. 3, Table 2a, b). In particular, a large cluster including precuneus and paracentral lobule with a right side dominance was found in the medial parietal region $(8068$ voxels for $\mathrm{ABM}<$ rest \& semantic memory < rest, 8078 voxels for $\mathrm{ABM}<$ rest \& number counting < rest), and it situated dorsally to the cluster in the medial parietal area which showed the overlap between greater activation during $\mathrm{ABM}$ and less activation during semantic memory or number counting (Fig. 3).

ABM-specific activation, which was defined as the overlap between the contrast of $\mathrm{ABM}$ vs. rest and the contrast of
$\mathrm{ABM}$ vs. semantic memory, was found in the left-lateralized brain regions including posterior cingulate gyrus/retrosplenial cortex, dorsal premotor area, ventromedial PFC, precentral gyrus, inferior parietal lobule, middle temporal gyrus, superior temporal gyrus, and frontopolar cortex (Fig. 4, Table 3). All of them except dorsal premotor area and middle temporal gyrus fell within the areas that showed less activation during number counting (compare Fig. 3 and Fig. 4). Contrast effect across conditions (ABM, semantic memory, and number counting) at the voxel of peak activation is shown in Fig. (5). All of the ABM-specific activation except for dorsal premotor area and middle temporal gyrus showed large negative contrast effect during number counting, sug- 
Table 1. Greater Activation During ABM Overlapped with Less Activation During SEM and NC

(a) $($ ABM $>$ REST) \& $($ SEM $<$ REST)

\begin{tabular}{|c|c|c|c|c|c|c|c|}
\hline Anatomical area & BA & side & $\mathbf{x}$ & $\mathbf{y}$ & $\mathbf{z}$ & z score & voxels \\
\hline Posterior cingulate gyrus & 31 & $\mathrm{~L}$ & -10 & -54 & 22 & 5.6 & $284^{\mathrm{a}}$ \\
\hline Precuneus & 7 & $\mathrm{~L}$ & -6 & -64 & 26 & 4.3 & $284^{\mathrm{a}}$ \\
\hline Ventromdial PFC & 11 & $\mathrm{~L}$ & -6 & 50 & -10 & 4.2 & 116 \\
\hline Inferior parietal lobule & 39 & $\mathrm{~L}$ & -44 & -64 & 28 & 3.1 & $80^{\mathrm{b}}$ \\
\hline
\end{tabular}

(b) $($ ABM $>$ REST $) \&($ NC $<$ REST $)$

\begin{tabular}{|c|c|c|c|c|c|c|c|}
\hline Anatomical area & BA & side & $\mathbf{x}$ & $\mathbf{y}$ & $\mathbf{z}$ & z score & voxels \\
\hline \multirow{2}{*}{ Posterior cingulate gyrus } & 30 & $\mathrm{~L}$ & -8 & -56 & 16 & Inf & $2748^{\mathrm{a}}$ \\
\hline & 29 & $\mathrm{R}$ & 10 & -46 & 4 & 5.6 & $2748^{\mathrm{a}}$ \\
\hline Premotor area & 8 & $\mathrm{~L}$ & -26 & 24 & 48 & 5.6 & 635 \\
\hline Hippocampal region & 27 & $\mathrm{~L}$ & -24 & -22 & -8 & 4.4 & 312 \\
\hline Inferior parietal lobule & 40 & $\mathrm{~L}$ & -38 & -68 & 38 & 4.0 & 173 \\
\hline Middle temporal gyus & 21 & $\mathrm{~L}$ & -54 & -38 & -6 & 4.0 & 81 \\
\hline Inferior frontal gyrus & 47 & $\mathrm{~L}$ & -40 & 34 & -10 & 3.7 & 220 \\
\hline \multirow{3}{*}{$\begin{array}{l}\text { Lingual gyrus } \\
\text { Inferior temporal gyrus }\end{array}$} & 8 & $\mathrm{~L}$ & -4 & -86 & -8 & 3.4 & 24 \\
\hline & 8 & $\mathrm{R}$ & 28 & 18 & 46 & 3.8 & 33 \\
\hline & 21 & $\mathrm{~L}$ & -62 & -10 & -18 & 3.4 & 20 \\
\hline
\end{tabular}

Voxels labelled by superscript suffix of the identical alphabet belong to one identical cluster.

gesting that most of the ABM-specific activation were located within the DMN.

\section{DISCUSSION}

There was a relatively large degree of overlap between greater activation during $\mathrm{ABM}$ and the DMN which was delineated by less activation during number counting. Extensive part of less activation during ABM also overlapped with less activation during number counting. These results show that both greater activation and less activation during ABM considerably overlapped with the DMN, suggesting that DMN is constituted of heterogeneous components with respect to ABM. The overlap between greater activation during $\mathrm{ABM}$ and less activation during number counting was found in the areas including the posterior cingulate gyrus, ventromedial PFC, hippocampal region, and inferior parietal lobule, suggesting that these regions are the DMN components associated with ABM. On the other hand, the overlap between less activation during $\mathrm{ABM}$ and less activation during number counting was found in the areas including the paracentral lobule extending into the precuneus and into the pre/post central gyrus (M1/S1), inferior parietal lobule, posterior insula extending into the superior temporal gyrus, most of which were also deactivated during semantic memory. Although the function subserved by these regions is unclear, they may represent the DMN component which is deactivated during any sorts of explicit task involving stimulus-oriented cognitive processes. This possibility can be reinforced by future studies showing that these regions also show less activation during other tasks which are known to increase activities of the DMN such as social cognition $[14,15,16]$ and imagining the thought and feeling of others (theory of mind) [14]. Alternately, MI/SI and posterior insular, in particular, might be related to processing of interoceptive sensory information such as somatic, proprioceptive, and visceral sense [22]. Deactivation of the DMN including these areas during discomfort and unpleasant gastric extension was reported in a recent study, which was interpreted as an adaptive 
Table 2. Less Activation During ABM Overlapped with Less Activation During SEM and NC

(a) $($ ABM $<$ REST) \& $($ SEM $<$ REST)

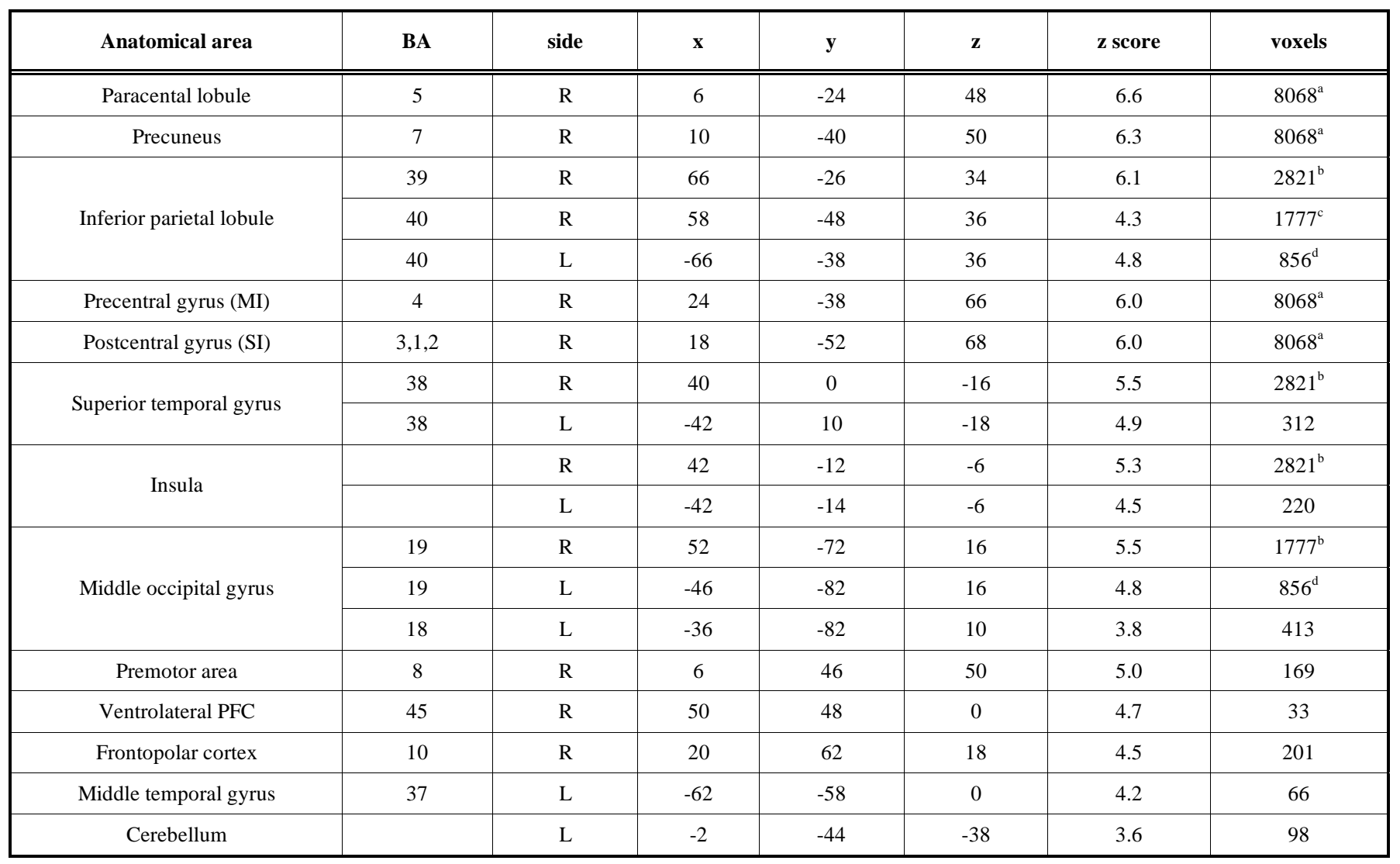

(b) $($ ABM $<$ REST) \& $($ NC $<$ REST)

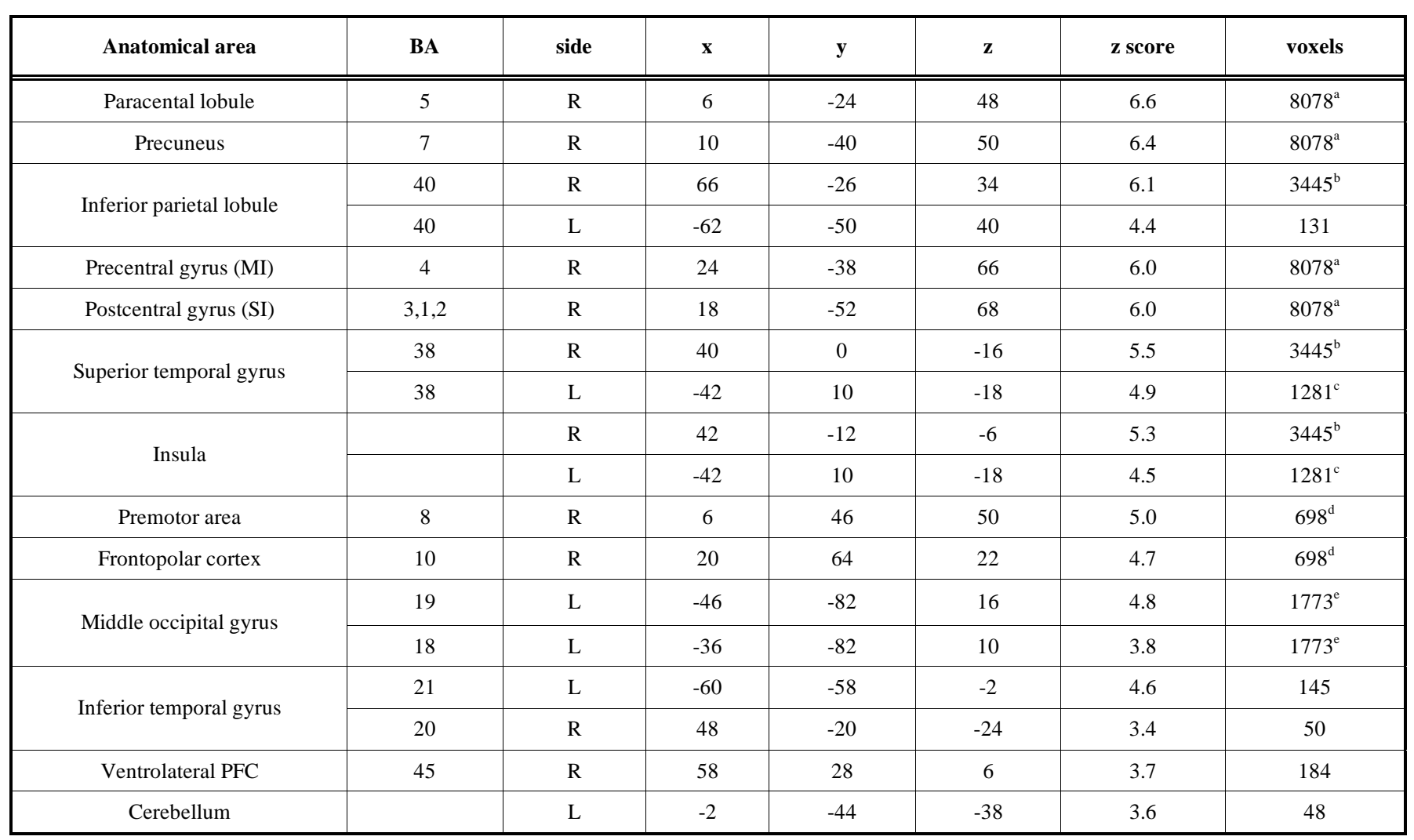

Voxels labelled by superscript suffix of the identical alphabet belong to one identical cluster. 


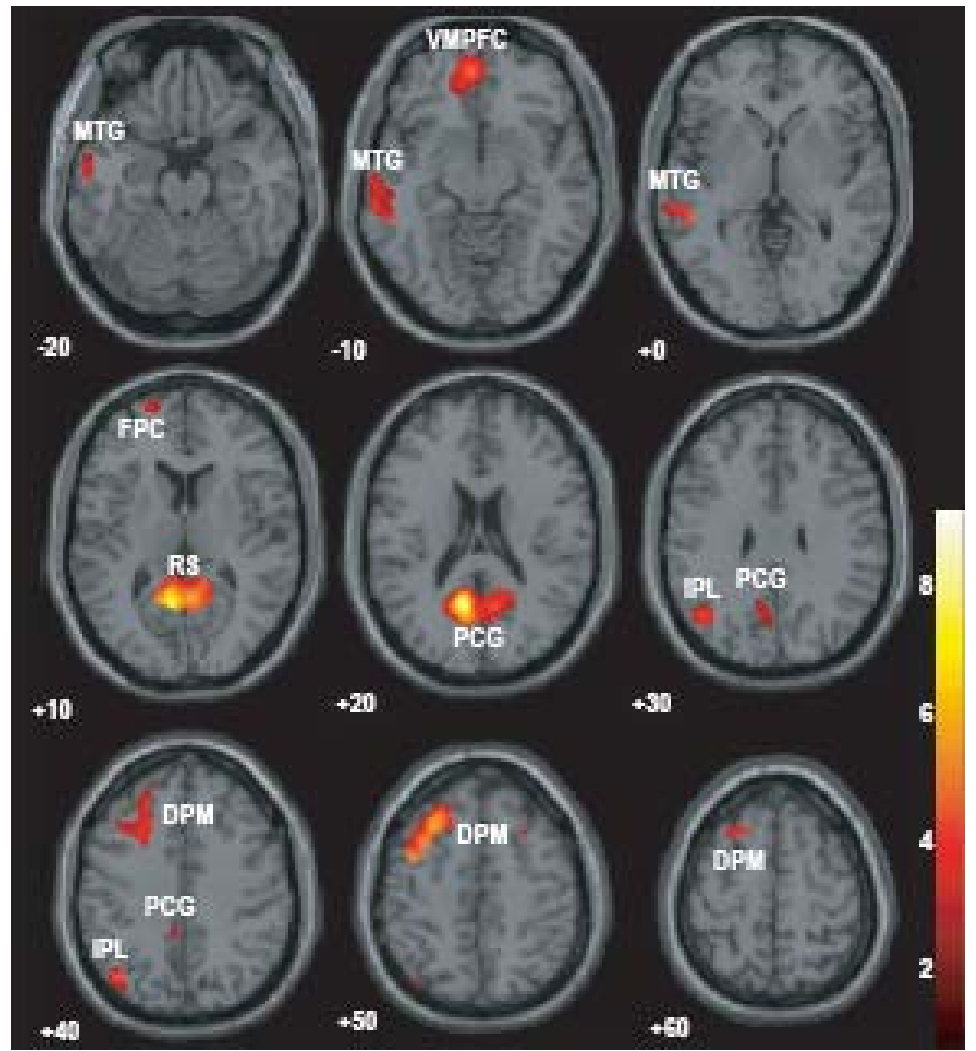

Fig. (4). The overlap of the contrast of ABM > REST and the contrast of ABM > SEM, superimposed on the axial images of the MNI T1 template. FPC, frontopolar cortex; DPM, dorsal premotor area; IPL, inferior parietal lobule; MTG, middle temporal gyrus; RS, retrosplenial cortex; PCG, posterior cingulate gyrus; STG, superior temporal gyrus; and VMPFC, ventro-medial prefrontal cortex.

Table 3. ABM-Specific Areas (ABM > REST \& ABM > SEM)

\begin{tabular}{|c|c|c|c|c|c|c|c|}
\hline Anatomical area & BA & side & $\mathbf{x}$ & $\mathbf{y}$ & $\mathbf{z}$ & z score & voxels \\
\hline \multirow{2}{*}{ Posterior cingulate gyrus } & 30 & $\mathrm{~L}$ & -8 & -56 & 16 & Inf & $1754^{\mathrm{a}}$ \\
\hline & 30 & $\mathrm{R}$ & 6 & -54 & 10 & 5.7 & $1754^{\mathrm{a}}$ \\
\hline Retrosplenial cortex & 29 & $\mathrm{R}$ & 10 & -46 & 4 & 5.6 & $1754^{\mathrm{a}}$ \\
\hline \multirow{2}{*}{ Dorsal premotor area } & 8 & $\mathrm{~L}$ & -38 & 2 & 54 & 6.0 & 1163 \\
\hline & 8 & $\mathrm{R}$ & 30 & 16 & 46 & 3.6 & 34 \\
\hline Ventromedial PFC & 11 & $\mathrm{~L}$ & -4 & 52 & -14 & 5.1 & 496 \\
\hline Superior temporal gyrus & 38 & $\mathrm{~L}$ & -50 & 20 & -20 & 4.1 & 30 \\
\hline Frontopolar cortex & 10 & $\mathrm{~L}$ & -22 & 62 & 10 & 3.3 & 44 \\
\hline
\end{tabular}

Voxels labelled by superscript suffix of the identical alphabet belong to one identical cluster. 

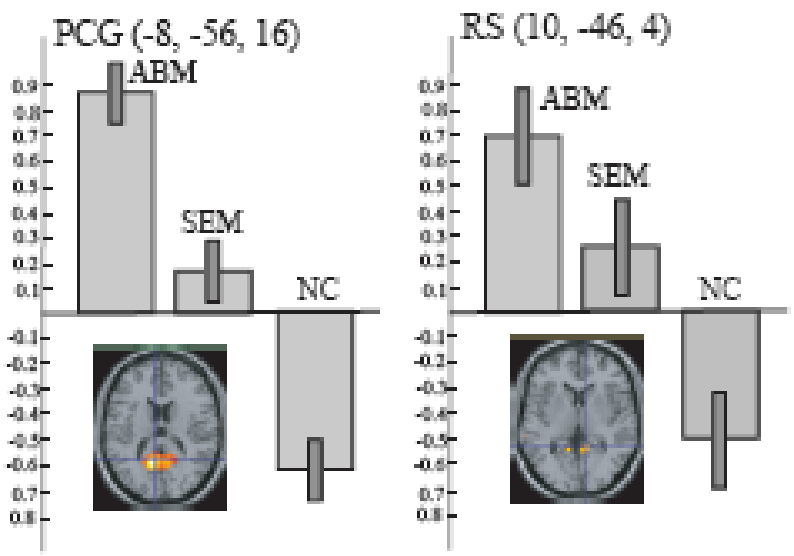

$\mathrm{DPM}(-38,2,54)$
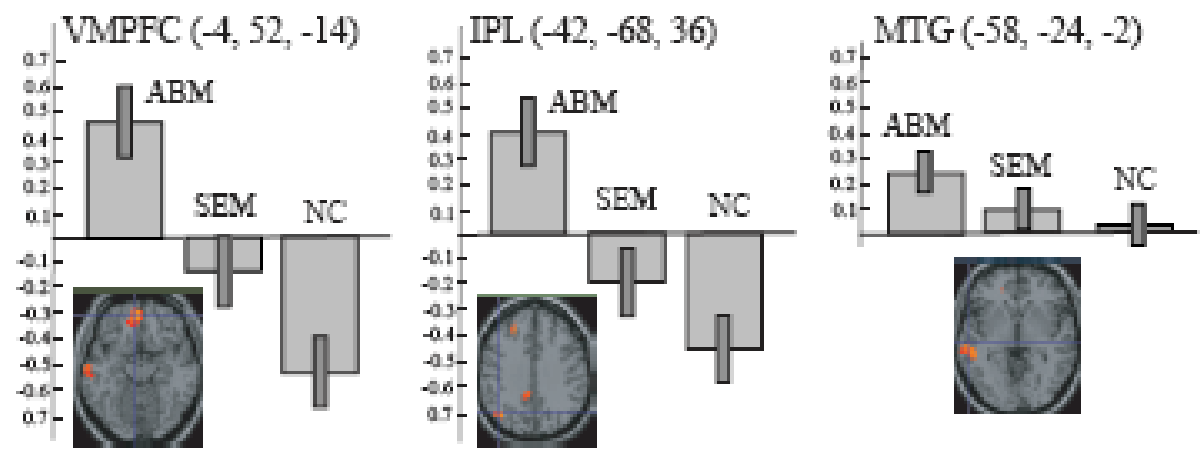

Fig. (5). The contrast effect of each task condition (ABM, SEM, and NC) compared to REST in the local maxima for the overlap between AMB > REST and ABM > SEM, which correspond to the intersection of lines in the lower panels. Bars represent the limit of the $90 \%$ confidence interval around the mean. DPM, dorsal premotor area; IPL, inferior parietal lobule; MTG, middle temporal gyrus; RS, retrosplenial cortex; PCG, posterior cingulate gyrus; and VMPFC, ventro-medial prefrontal cortex.

antinociceptive response or shift from exteroceptive to interoceptive sensory processing [23]. A recent functional connectivity analysis also indicated that anterior dorsal precuneus exhibits functional connectivity with sensorimotor region and insula [24]. The cluster in the medial parietal region including the precuneus and paracentral lobule which is a component of the overlap between less activation during $\mathrm{ABM}$ and less activation during number counting was located dorsally to the cluster in the medial parietal region including the posterior cingulate gyrus and retrosplenial cortex which is a component of the overlap between greater activation during $\mathrm{ABM}$ and less activation during number counting. This may suggest that medial parietal region of the DMN is functionally dissociated into the dorsal part (anterior part of the precuneus and paracentral lobule) and ventral part (posterior cingulate gyrus and retrosplenial cortex), but future studies are necessary in order to elucidate the behavioral and physiological meaning of this functional dissociation.

In the present study, ABM-specific activation was defined as the overlap between the contrast of ABM vs. rest and the contrast of $A B M$ vs. semantic memory. We believe that this definition delineated the ABM-specific region more reliably than previous studies, which investigated the brain activation during $\mathrm{ABM}$ task relative to one reference task such as rest or retrieval of semantic memory, due to the reason described above. The ABM-specific activation in the present study was found in the left-lateralized areas includ- ing the posterior cingulate gyrus/retrosplenial cortex, dorsal premotor area, ventromedial PFC, precentral gyrus, inferior parietal lobule, middle temporal gyrus, superior temporal gyrus, and frontopolar cortex, which is consistent with previous studies except for the absence of the hippocampal activation [2, 27, 28, 29]. A recent review of event-related fMRI and ERP studies showed that the inferior parietal cortex, especially in the left hemisphere, is associated with successful recollection [30]. On the other hand, lesion study indicated that right-sided frontal and temporal lobe might make a greater contribution to ABM [31]. Electrophysiological study showed that the ABM-related neuronal responses emerged in left frontal at early phase of memory generation and shifted toward the right and posterior direction at late phase when memory was hold [1, 32]. It was also suggested that autobiographical memory associated with a cue word may initially recruit left temporal structures, but later may be more dependent on the right temporal lobe [29]. Although most studies permit participants to fully recollect an event and rate the amount of details after the recollection, the present study instructed participants to produce autobiographical memory as much as possible and press a button successively when one appropriate item came to mind. Therefore, the present task involved early phase of ABM and did not involve later phase such as maintenance and evaluation of the memory trace. This is the probable reason why ABM-specific activation in the present study was left-lateralized. Most of these ABMspecific activation fell within the areas which showed less 
activation during number counting relative to rest, suggesting that areas of ABM-specific activation are parts of the DMN, although large areas of greater activation during ABM were not overlapped with less activation during number counting and therefore they were present outside the DMN (cf. large area shown red colour in Fig. 3). Activation during $\mathrm{ABM}$ outside of the DMN in the present study included the frontoparietal control network such as the rostrolateral PFC, anterior insula, and dorsal anterior cingulate cortex [25]. Spreng et al. [26] recently showed that this frontoparietal control network flexibly couples its activity with either the DMN or dorsal attention network in support of goal-directed cognition. These areas will be engaged to aid in the retrieval process and updating the items being counted in the memory in the present study.

Activation of the hippocampal region did not emerge as belonging to the network unique to ABM. This is because hippocampal region was not activated in the contrast of ABM vs. semantic memory (data not shown), although posterior region of the hippocampus showed greater activation in the contrast of ABM vs. rest (see Fig. 3, z = 10). Recent imaging studies of healthy individuals have reported hippocampal activation in both episodic and semantic retrieval [20, 33]. In particular, Ryan et al. [20] showed that hippocampal activation during semantic memory retrieval which is not contaminated by autobiographical content was similar to that during autobiographical memory retrieval. These neuroimaging findings, which showed that the degree of neuronal responses in the hippocampal area was similar between $\mathrm{ABM}$ and semantic memory, apparently contradicts with the clinical finding that hippocampal injury causes disturbances of episodic/autobiographical memory but not of semantic memory [34]. Although some patients with hippocampal lesion may have significant deficits in semantic memory [3537], it is a general consensus that semantic memory disturbance is much less severe than autobiographical memory loss in hippocampal damage. This discrepancy between neuroimaging finding and the clinical finding can be reconciled when we consider that the hippocampal region is a key node or hub region for ABM but not for semantic memory and therefore hippocampal damage causes disturbance of $\mathrm{ABM}$ sparing semantic memory. Even though the hippocampal activation is similar between ABM and SEM, the hippocampal region appears to be anatomically pivotal only for ABM. Similar suggestion regarding the discrepancy between functional imaging studies and lesion studies was discussed by the concept of degeneracy which proposes a limited number of specialized systems for the same function [38].

The present study has several limitations. First, subject's responses were simple button press, and therefore the appropriateness of the responses could not be fully guaranteed. Second, different categories were used between ABM and semantic memory and therefore those retrieval tasks were not well matched. Third, since there were only 6 trials whose duration was $96 \mathrm{~s}$ in total for each task condition, statistical power will be relatively small due to a rather small number of samples. For example, the finding that the hippocampal region did not emerge as belonging to $\mathrm{ABM}$-specific network should be interpreted with caution.

\section{CONCLUSION}

We delineated the overlap between the DMN and greater/less activation during $\mathrm{ABM}$ in order to reveal components of the DMN which are related to ABM and those, which are unrelated to ABM. Less activation as well as greater activation during ABM overlapped considerably with the DMN, suggesting that the DMN is functionally segregated to the regions which are associated with ABM, such as posterior cingulate gyrus and retrosplenial cortex, and those which are unrelated to ABM, such as anterior precuneus and paracentral lobule. We further localized ABMspecific regions by extracting the overlap between activation during $\mathrm{ABM}$ relative to rest, and that relative to semantic memory. They were observed in the left-lateralized brain regions including posterior cingulate gyrus/retrosplenial cortex, dorsal premotor area, left ventromedial prefrontal cortex, and middle temporal gyrus, and most of them were located within the DMN.

\section{REFERENCES}

[1] Conway MA, Pleydell-Pearce CW. The construction of autobiographical memories in the self-memory system. Psychol Rev 2000; 107: 261-88.

[2] Maguire EA. Neuroimaging studies of autobiographical event memory. Philos Trans R Soc Lond B Biol Sci 2001; 356: 1441-51.

[3] Svoboda E, McKinnon MC, Levine B. The functional neuroanatomy of autobiographical memory: A meta-analysis. Neuropsychologia 2006; 44: 2189-208.

[4] Mazoyer B, Zago L, Mellet E, et al. Cortical networks for working memory and executive functions sustain the conscious resting state in man. Brain Res Bull 2001; 54: 287-98.

[5] Raichle ME, MacLeod AM, Snyder AZ, et al. A default mode of brain function. Proc Natl Acad Sci USA 2001; 98, 676-82.

[6] Greicius MD, Krasnow B, Rriss A, Menon V. Functional connectivity in the resting brain: a network analysis of the default mode hypothesis. Proc Natl Acad Sci USA 2003; 100: 253-8.

[7] Mason MF, Norton MI, Van Horn JD, et al. Wandering Minds: The default network and stimulus-independent thought. Science 2007; 315: 393-5.

[8] Buckner RL, Andrews-Hanna JR, Schacter DL. The brain's default network: anatomy, function, and relevance to disease. Ann NY Acad Sci 2008; 1124: 1-38.

[9] Raichle ME, Mintun MA. Brain work and brain imaging. Annu Rev Neurosci 2006; 29: 449-76.

[10] Raichle ME, Snyder AZ. A default mode of brain function: a brief history of an evolving idea. NeuroImage 2007; 37: 1083-90.

[11] Carhart-Harris RL, Friston KJ. The default-mode, ego-functions and free-energy: a neurobiological account of Freudian ideas. Brain 2010; 133: 1265-83.

[12] Andreasen NC, O'Leary DS, Cizadlo T, et al. Remembering the past: two facets of episodic memor explored with positron emission tomography. Am J Psychiatry 1995; 152: 1576-85.

[13] Vincent JL, Snyder A., Fox MD, et al. Coherent spontaneous activity identifies a hippocampal-parietal memory network. J Neurophysiol 2006; 96: 3517-31.

[14] Spreng RN, Grady CL. Patterns of brain activity supporting autobiographical memory, prospection, and theory of mind, and their relationship to the default mode network. J Cogn Neurosci 2009; 22: 1112-23.

[15] Spreng RN, Mar RA, Kim AS. The common neural basis of autobiographical memory, prospection, navigation, theory of mind, and the default mode: A quantitative meta-analysis. J Cogn Neurosci 2009; 21: 489-510.

[16] Schilbach L, Eickhoff SB, Rotarska-Jagiela A, et al. Minds at rest? Social cognition as the default mode of cognizing and its putative relationship to the "default system" of the brain. Conscious Cogn 2008; 17: 457-67.

[17] Laird AR, Eickhoff SB, Li K, Robin DA, Glahn DC, Fox PT. Investigating the functional heterogeneity of the default mode network using coordinate-based meta-analytic modeling. J Neurosci 2009; 29:14496-505. 
[18] Uddin LQ, Kelly AM, Biswal BB, Xavier Castellanos F, Milham MP. Functional connectivity of default mode network components: correlation, anticorrelation, and causality. Hum Brain Mapp 2009; 30: 625-37.

[19] Tulving E. Episodic and semantic memory. In Tulving E.\& Donaldson W. (Eds.) Organization of memory. Oxford, England: Academic Press, 1972.

[20] Ryan L, Cox C, Hayes SM, Nadel L. Hippocampal activation during episodic and semantic memory retrieval: comparing category production and category cued recall. Neuropsychologia 2008; 46: 2109-21.

[21] Oldfield RC. The assessment and analysis of handedness: the Edinburgh inventory. Neuropsychology 1971; 9: 97-113.

[22] Craig AD. How do you feel-now? The anterior insula and human awareness, Nature Rev Neurosci 2009; 10: 59-70.

[23] Van Oudenhove L, Vandenberghe J, Dupont P, et al. Cortical deactivations during gastric fundus distension in health: visceral pain-specific response or attenuation of 'default mode' brain function? A H2 15O-PET study. Neurogastroenterol Motil 2009; 21: 259-71.

[24] Margulies DS, Vincent JL, Kelly C, et al. Precuneus shares intrinsic functional architecture in humans and monkeys. Proc Natl Acad Sci USA 2009; 106: 20069-74.

[25] Vincent JL, Kahn I, Snyder AZ, Raichle ME, Buckner RL. Evidence for a frontoparietal control system reveaked by intrinsic functional connectivity. J Neurophysiol 2008; 100: 3328-42.

[26] Spreng RN, Stevens WD, Chamberlain JP, Gilmore, AW, Schacter DL. Default network activity, coupled with the frontoparietal control network, supports goal-directed cognition. NeuroImage 2010; 53: 303-17.
[27] Conway MA, Pleydell-Pearce CW, Whitecross S, Sharpe H. Brain imaging autobiographical memory. Psychol Learn Motiv 2002; 41: 229-64.

[28] Gilboa A. Autobiographical and episodic memory-one and the same? Evidence from prefrontal activation in neuroimaging studies. Neuropsychologia 2004; 42: 1336-49.

[29] Graham KS, Lee AC, Brett M, Patterson K. The neural basis of autobiographical and semantic memory: new evidence from three PET studies. Cogn Affect Behav Neurosci 2003; 3: 234-54.

[30] Vilberg KL, Rugg MD. Memory retrieval and the parietal cortex: A review of evidence from a dual-process perspective. Neuropsychologia 2008; 46: 1781-99.

[31] Kopelman MD, Stanhope N, Kingsley D. Retrograde amnesia in patients with diencephalic, temporal lobe or frontal lesions. Neuropsychologia 1999; 37: 939-58.

[32] Conway MA. The neuroanatomy of autobiographical memory: a slow cortical potential study of autobiographical memory retrieval. J Mem Lang 2001; 45: 493-524.

[33] Burianova H, Grady CL. Common and unique activations in autobiographical, episodic, and semantic retrieval. J Cogn Neurosci 2007; 19: 1520-34.

[34] Moscovitch M, Nadel L, Winocur G, Gilboa A, Rosenbaum RS. The cognitive neuroscience of remote episodic, semantic and spatial memory. Curr Opin Neurobiol 2006; 16: 179-90.

[35] Luo J, Niki K. Role of medial temporal lobe in extensive retrieval of task-related knowledge. Hippocampus 2002; 12: 487-94.

[36] Manns JR, Hopkins RO, Squire LR. Semantic memory and the human hippocampus. Neuron 2003; 38: 127-33.

[37] Squire LR, Stark CE, Clark RE. The medial temporal lobe. Annu Rev Neurosci 2004; 27: 279-306.

[38] Price CJ, Friston KJ. Degeneracy and cognitive anatomy. Trends Cogn Sci 2002; 6: 416-21.

Received: September 21, 2010

Revised: November 04, 2010

Accepted: November 05, 2010

(C) Ino et al.; Licensee Bentham Open.

This is an open access article licensed under the terms of the Creative Commons Attribution Non-Commercial License (http://creativecommons.org/licenses/by-nc/3.0/) which permits unrestricted, non-commercial use, distribution and reproduction in any medium, provided the work is properly cited. 